Théologiques

Théologiques

\title{
Le canon et ses suites : entre concept et figure
}

\section{Bill Readings}

Volume 1, numéro 2, octobre 1993

Les textes fondateurs. Entre autorité et liberté

URI : https://id.erudit.org/iderudit/602390ar

DOI : https://doi.org/10.7202/602390ar

Aller au sommaire du numéro

Éditeur(s)

Faculté de théologie de l'Université de Montréal

ISSN

1188-7109 (imprimé)

1492-1413 (numérique)

Découvrir la revue

Citer cet article

Readings, B. (1993). Le canon et ses suites : entre concept et figure.

Théologiques, 1(2), 39-62. https://doi.org/10.7202/602390ar
Résumé de l'article

II s'agit d'une étude du débat sur l'autorité du canon en études littéraires et religieuses aux États-Unis qui argumente, à partir de la lecture freudienne de la Bible dans Moïse et le monothéisme, en vue d'une approche du canon comme figure de l'état non originaire de la culture plutôt que comme objet déterminé par son contenu intrinsèque ou des contraintes externes. d'utilisation que vous pouvez consulter en ligne.

https://apropos.erudit.org/fr/usagers/politique-dutilisation/ 


\title{
Le canon et ses suites: entre concept et figure.
}

\author{
Prof. Bill READINGS \\ Département de littérature comparée \\ Université de Montréal
}

\section{RÉSUMÉ}

II s'agit d'une étude du débat sur l'autorité du canon en études littéraires et religieuses aux États-Unis qui argumente, à partir de la lecture freudienne de la Bible dans Moïse et le monothéisme, en vue d'une approche du canon comme figure de l'état non originaire de la culture plutôt que comme objet déterminé par son contenu intrinsèque ou des contraintes externes.

\section{Préambule 1}

II est plus que jamais question en études littéraires de l'autorité à accorder aux textes fondateurs qui forment les divers canons des littératures nationales. Nous croyons que, pour bien saisir les enjeux de ce débat, il convient de le comparer avec les discussions récentes sur l'autorité du canon biblique en études religieuses. Cette comparaison nous permettra de concevoir à nouveau le canon non pas comme une simple liste de textes qui posséderaient leur propre autorité, mais

1 Une version préliminaire de cette étude à été publiée en anglais dans le Journal of the American Academy of Religion 57/1. L'auteur remercie Luce Desmarais pour son aide précieuse dans la préparation de cet article. 
comme la figure dédoublée d'une autorité textuelle qui à la fois nécessite et délégitime l'acte de lire.

Il existe une raison historique précise pour laquelle ce débat continue d'être plus virulent dans les départements de littérature en Amérique du Nord. Le débat sur le canon littéraire est spécifiquement nord-américain parce que le canon incarne la possibilité de la continuité historique, dans un corpus de textes littéraires conçu comme un ensemble d'objets discontinus parce que autonomes. Le formalisme de la New Criticism fait de l'objet d'art une entité autonome, sans histoire, ce qui constitue un gain démocratique appréciable dans une société d'immigrants : l'oeuvre peut être abordée par n'importe qui, sans connaissance préalable de l'histoire ou de la tradition. L'historicité de l'oeuvre littéraire en Amérique du Nord est garantie par son inclusion dans le canon: c'est la raison du succès commercial des anthologies de littérature. La Constitution américaine, en tant que texte fondateur d'une démocratie républicaine formée par des vagues successives d'immigration, repose sur la volonté du peuple et non sur la tradition. Mais la tradition, refoulée par la Déclaration d'Indépendance, revient sous la forme d'une volonté populaire déterminant ce qui doit constituer cette tradition: c'est-à-dire sous la forme du canon esthétique. Le canon représente le choix, et non l'expérience, d'une tradition. Le livre de Hirsch, Cultural Literacy, est tout à fait représentatif de ce fonctionnement: l'identité culturelle comme décompte des faits à connaître plutôt que comme tradition historique -- approche qui, incidemment, facilite la préparation de ces tests standardisés sur l'identité culturelle que sont les SAT et les GRE de littérature 2 . L'idée du canon sert à résoudre la contradiction entre ethnicité historique et volonté républicaine, dans la mesure où létablissement du canon est conçu comme un exercice du libre-arbitre rationnel du peuple américain par lequel ce dernier "choisit" sa propre ethnicité historique. Ce que vivent aujourd'hui les départements de littérature aux États-Unis n'est pas tant une révision du canon qu'une crise de la fonction du canon. II s'agit peut-être d'un des signes les plus clairs du déclin de l'idée de l'état-nation: le canon ne parvient plus à englober, sous l'appellation de "culture", la volonté populaire et une fiction ethnique. On peut ainsi considérer la mise en question du canon comme symptôme de la condition crépusculaire de l'état-nation moderne.

2 SAT : Scholastic Aptitude Test; GRE : Graduate Record Examination. Ces examens sont obligatoires pour poursuivre des ćtudes supérieures aux États-Unis. 
La réflexion universitaire sur le canon, en études littéraires comme en études religieuses, prend plusieurs formes. Les défenseurs du concept du canon veulent, à divers titres, lui attribuer une autorité intrinsèque: les conservateurs défendent le caractère clos du canon traditionnel alors que les libéraux affirment que le canon est ouvert et nécessairement sujet à révision. Que ses défenseurs le définissent de manière idéaliste (le canon comme incarnation de valeurs ou de vérités transcendantes, par analogie avec la voix divine) ou de manière "historiciste" (le canon comme grand organisme formé par la sédimentation des textes d'une même culture, formant un tout plus grand que la somme de ses parties, l'identité collective), dans les deux cas, la notion de canon comporte toujours une autorité essentielle.

Les adversaires du canon soutiennent, pour leur part, que le canon sert à promouvoir les valeurs d'une certaine logique culturelle qui lui est extrinsèque : la canonisation serait l'établissement d'une orthodoxie opprimante, une opération idéologique basée sur l'autorité tendancieuse du prestige littéraire ou de l'identité culturelle. Selon ce dernier argument, toute défense du canon dans sa forme actuelle n'est rien d'autre qu'un refus entêté de considérer les forces extrinsèques qui ont modelé le canon.

\section{Le canon en littérature}

\subsection{Préserver le canon: la position de l'idéalisme conservateur}

Walter Jackson Bate, professeur en études littéraires à Harvard, ainsi que d'autres héritiers de l'impérialisme culturel victorien, voient dans le canon littéraire anglais l'incarnation de valeurs transcendantes authentiques. C'est pourquoi Bate taxe d'insignifiance des littératures aussi peu canoniques que la littérature chicano ou noire. La position de l'idéalisme conservateur est aujourd'hui largement discréditée sur la base d'une argumentation extrinsèque, par les critiques qui font valoir que cette position produit une version singulièrement blanche, mâle et bourgeoise de la transcendance 3 . Cette critique ébranle les affirmations de l'idéalisme conservateur en montrant que la valeur soidisant intrinsèque du canon n'est pas elle-même canonique.

3 Le travail de Gayatri Chakravorty Spivak est exemplaire de cette critique. Elle parle de «current and continued subalternization of so-called 'third world' literatures» (SPIVAK 1987: 241). 
Mais l'affirmation de la valeur intrinsèque du canon est problématique par elle-même. Poussée à l'extrême, la logique de l'argument à l'effet que le canon fait autorité parce qu'il incarne un idéal transcendant de "valeur littéraire" prive en fait le canon de toute autorité intrinsèque. Dans cette optique, le canon ne devient qu'un aidemémoire permettant de découvrir un idéal de valeur littéraire qui serait elle-même extrinsèque au canon. Cette conception du canon ne permet pas de maintenir une division entre intériorité et extériorité du canon, que sa forme close semblait pourtant garantir.

\subsection{L'orthodoxie du canon: la position de l'«uni- culturalisme" conservateur}

On s'accorde habituellement à voir dans le texte de T.S. Eliot, "Tradition and the Individual Talent", l'expression classique de la position du conservatisme culturel en études littéraires. Dans cet article, une notion exclusiviste du canon sert à définir l'identité collective anglaise ... le "native element" (1964: 24) que Eliot, dans The Use of Poetry and the Use of Criticism, admirait chez Dryden. John Guillory a fait de la tradition de "Tradition and the Individual Talent" une critique systématique sous le titre de "Orthodoxy and the Literary Elite". L'affirmation d'une identité culturelle, mis à part l'argument moral précité contre l'impérialisme culturel, est entachée d'un vice logique. En effet, l'autorité qui doit fonder la culture (l'autorité du canon) est elle-même un effet du canon qu'elle prétend fonder. Ce cercle vicieux est analogue à l'aporie que présente le modèle contractuel de l'État, selon lequel la constitution est garantie par des sujets qui sont précisément habilités à ce faire en vertu de la constitution qu'ils doivent garantir...

\subsection{Repenser le canon : la position du révisionnisme culturel}

Les critiques du canon qui réclament l'inclusion de textes non canoniques dans le canon, ou la formation de canons alternatifs, partagent avec les tenants du conservatisme culturel une même idée du canon comme totalité organique qui définit et représente la culture. L'autorité de cette représentation transcende celle de chacun des textes qu'elle englobe -- d'où le fait que l'imprimatur que représente l'inclusion dans le canon est précisément recherché. Lillian S. Robinson décrit bien le revirement qui s'est produit chez certaines féministes littéraires, qui 
sont passées des revendications pour la simple inclusion des femmes dans le canon traditionnel au développement d'un canon distinct. Dans la même veine, un "révisionniste" comme Lawrence Lipking a pu saluer les nouvelles avenues ouvertes par une nouvelles poétique féminine ${ }^{4}$. Dans cette lignée, l'exemple le plus marquant d'un canon féministe séparé est la Norton Anthology of Literature by Women de Gilbert et Gubar. Dans le même mouvement, Arnold Krupat revendique de manière convaincante l'inclusion de la littérature autochtone dans le canon littéraire américain. Dans tous ces exemples, il ne s'agit toujours cependant que d'un canon dont le contenu aurait une valeur intrinsèque. La discussion se limite à déterminer quelles oeuvres devraient y être incluses. Dans ce raisonnement, il s'agit de protéger à l'avenir le canon contre des facteurs extrinsèques (le sexisme et l'ethnocentrisme) qui ont marqué sa formation. De telles critiques renforcent en fait la conception canonique de la littérature.

\subsection{Normaliser un canon ouvert: la position de l'idéalisme libéral}

Une réplique libérale aux critiques du canon est donnée par Charles Altieri. Ce dernier tient compte des arguments extrinsèques et conçoit lui aussi le canon comme idéologiquement déterminé. Le canon retient toutefois, pour Altieri, une certaine autorité intrinsèque. Mais il désire cependant éviter de tomber dans un impérialisme primaire du genre "notre idéologie vaut mieux que la vôtre". Pour lui, la consécration canonique ne fonctionne pas de manière positive, pour renforcer la culture (comme chez T.S. Eliot), mais plutôt à la manière d'un idéal négatif. II y a donc pour lui, dans le canon, une transcendance négative inhérente. Le canon n'a pas pour fonction de dissimuler l'idéologie sous le masque d'une vérité transcendante, anhistorique, mais permet plutôt de saisir l'influence de cette idéologie dans la formation de notre présent. Le canon est ouvert, et non clos, parce que la quintessence de notre expérience historique qu'il incarne est un défi auquel nous devons maintenant répondre dans notre diversité. La tentative d'Altieri de présenter le canon comme dépositaire d'idéaux humanistes agissant négativement plutôt que positivement, nous forçant à nous y mesurer plutôt que confortant notre suffisance, est la défense la plus ingénieuse du canon en tant que concept. Selon lui, l'autorité du canon découle de sa capacité de produire des énoncés tant descriptifs que normatifs [c'està-dire prescriptifs] (Altieri 1983:40). Le canon idéal proposé par

4 «[A] women's poetics [that] gives us new ways of reading» (LIPKING1983:79). 
Altieri doit à la fois incarner (descriptivement) le passé et ordonner (prescriptivement) le futur : sa fonction est de "transmit the past as a set of challenges and models" (Altieri 1983:40). Mais, même si Altieri tient compte de la critique extrinsèque du canon, dans la mesure où ce dernier est compris comme un concept qui normalise la définition de la culture, le fonctionnement du canon -- même négatif .- ne peut que reproduire l'injustice même qu'il condamne en reconnaissant la validité des critiques extrinsèques. L'injustice est inévitable parce qu'Altieri tente de faire du canon un concept pouvant normaliser le passage d'un état d'énonciation descriptif à un état prescriptif. Ceci tombe sous le coup de la thèse de Jean-François Lyotard dans $A u$ juste, à l'effet que l'injustice résulte précisément d'une telle confusion entre les prescriptions et les descriptions. La Terreur est engendrée par toute tentative de fonder la justice d'un jugement éthique par référence à un ordre des choses représentable (un [ensemble d'] énoncé[s] descriptif[s]). Vouloir justifier la justice, prétendre fonder la prescription éthique en faisant appel à la catégorie descriptive -- et ce, que le canon fonctionne positivement ou négativement -- est fondamentalement injuste. La justice qui maintient l'hétérogénéité des prescriptions éthiques et des descriptions n'est donc pas elle-même un savoir (un [des] énoncé[s] descriptif[s]). C'est une figure, une utilisation du langage qui, par sa constitution même, ne peut être retraduite en énoncés descriptifs littéraux. Or le canon est justement une telle figure.

\subsection{Démystifier le canon: la position extrinsèque}

À l'encontre des défenses du canon fondées sur l'assertion de sa valeur intrinsèque, peuvent être opposées une série d'interprétations sociologiques ou historiques du canon lui enlevant toute autorité intrinsèque. D'une position d'extériorité scientifique, ces interprétations exposent les dimensions historique et idéologique latentes qui motivent la forme et l'autorité du canon. De telles interprétations, que nous avons appelées extrinsèques, remettent en question l'idée même du canon comme étant élitiste, une superstition archaïque et répressive. Dans cette argumentation, le canon est critiqué à cause de l'exclusion impérialiste qu'il fait de certains groupes et du silence qu'il impose à leur voix. Abdul R. JanMohamed parle de "tentatives par des canonisateurs ethnocentriques de langue anglaise et d'autres langues (occidentales) ainsi que des départements de littérature d'ignorer la 
culture et l'art du Tiers-Monde..."5. Pour Richard Ohmann, le canon est un "processus hégémonique", argument sous-tendu par l'axiome soidisant universel que "la valeur esthétique découle de la lutte des classes"6. De même, Christine Froula avance l'idée que le féminisme pose non seulement un défi radical à "l'histoire et la politique de la constitution du canon", mais aussi à "l'idée (et à l'idéal) du canon»?. Selon ces critiques, le canon sert à légitimer des abus de pouvoir tout à fait extrinsèques au canon lui-même. Pour corriger celte imposture idéologique, il est nécessaire de se débarrasser de l'idée même du canon, jugée élitiste et répressive. Bien que cette critique puisse sembler radicale et sans appel -. des siècles d'injustices lui donnent effectivement une résonnance importante --, ce n'est toutefois pas la lecture la plus radicale qui puisse être faite du canon.

\section{Le canon en études religieuses}

Les deux modèles d'argumentation en présence au débat sur le canon littéraire, intrinsèque et extrinsèque, semblent structurer également le débat en études religieuses. Brevard Childs, doyen de la "critique canonique" aux États-Unis, est le plus énergique défenseur du canon organique, porteur d'une valeur intrinsèque. Dans le camp de la science canonique, se retrouvent plusieurs tentatives contraires d'expliquer le canon biblique sur des bases extrinsèques, à savoir: historique/textuelle (James Barr), sociologique (Gerald Bruns) et politique (Elaine Pagels). Childs pour sa part propose une lecture déterminée par le "contexte du canon", qui admet le poids historique du privilège accordé au texte canonique par-dessus les "événements ou expériences derrière le texte" en évitant cependant de tomber dans le fondamentalisme textuel d'une "méthode pré-critique qui se sentirait libre de traduire simplement tout énoncé en principe de doctrine authentique» 8 . L'insistance de Childs sur le dernier état du texte canonique fonctionnant comme contexte normatif de l'interprétation accorde une autorité intrinsèque aux Écritures d'une communauté

5

«... attempts by ethnocentric canonizers in English and other (Western) language and literature departments to ignore Third World culture and art ..." (JANMOHAMED 1985:85)

6 «... aesthetic value arises from class conflict» (OHMANN 1983:219).

7 «[Feminism not only poses a challenge to] the history and politics of canonformation ... [but also to] the idea (and ideal) of the canon» (FROULA 1983:323).

8 «[the textual fundamentalism of] a pre-critical method which could feel free to simply translate every statement into a principle of right doctrine» (CHILDS 1986:6). 
d'interprétation donnée, malgré la contingence historique de cette communauté elle-même. L'autorité intrinsèque que voient les fondamentalistes dans la parole divine est transposée par Childs dans l'autorité intrinsèque du canon, lien fondateur de la communauté d'interprétation. Même si Childs parle de "contexte" canonique, l'autorité intrinsèque du canon est préservée car elle se retrouve intacte dans la fonction fondatrice du canon.

Le développement de la critique historique de la Bible a donné lieu à trois grands genres d'études sur le canon reposant toutes sur un sens latent et la recherche de faits extrinsèques. Leur dénominateur commun est l'idée que la vérité du canon biblique est extrinsèque au canon luimême, que cette vérité soit historique (pour Freud), théologique (pour Barr) ou socio-politique (pour Bruns et Pagels). Malgré les réserves de James Barr au sujet de l'historicisme (BARR 1983:105) et de la scientificité (BARR 1983:112-4), celui-ci reconnaît ouvertement que son épistémologie prend racine dans le terreau des Lumières (BARR 1983:122-4). Barr défend une approche des Écritures qui exclut explicitement du canon le moment de sa formation. Le canon a ses origines dans l'histoire9. Les analyses littéraires, linguistiques, historiques et sociologiques qu'il propose portent sur le canon et visent à retrouver une vérité extra-canonique. À cet égard, Barr est effectivement, comme le soutient Childs, un héritier de la tradition de la critique historique de la Bible : son analyse tend à démystifier le canon et refuse de distinguer entre la Bible et d'autres sources de savoir. Ainsi il soutient que pour le christianisme biblique, "Les Écritures sont essentielles alors que le canon ne l'est pas»10. La vérité ou l'autorité théologique des Écritures découle de facteurs complètement extrinsèques à leur canonisation institutionnelle (BARR 1983: 101). De fait, l'institution du canon devient un mythe sous lequel se cache une réalité biblique cachée ${ }^{11}$. Chez Barr, la vérité décisive de la réalité

9 «[I]n thus taking its stand upon the canon as a complete entity, the church -- and especially the Protestant branch of it -- was actually taking its stand, to a considerable extent, outside the Bible rather than within it. To take completed scripture as the starting point was automatically to place the starting point outside the situation of biblical man» (BARR 1983:3). La formulation de ce passage montre clairement que la critique tolérante et sympathique de Barr à l'endroit de Childs et de ses disciples repose sur un schéma du canon en termes d'intériorité et d'extériorité qui est pris pour acquis par les parties au débat.

10 «Scripture is essential, but canon is not» (Bart 1983:63).

11 On peut voir un exemple de la remise en question produite par l'analyse de Barr dans sa description du canon du Nouveau Testament : 
biblique cachée a la même fonction que le contexte servant à déterminer l'intention de l'auteur dans la rédaction des Écritures ${ }^{12}$. Gerald Bruns a pour sa part produit une analyse extrinsèque purement sociologique dans laquelle le canon est démystifié au nom d'un fait social latent dans l'Écriture: "The power of the text is not intrinsic to it. On the contrary, the text draws its power from the situation in which it makes its unexpected appearance" (Bruns 1984: 466). La canonisation est donc un simple effet de pouvoir, une mystification idéologique produite par la domination d'un groupe social donné13. Bruns reprend à son compte l'analyse politique de Elaine Pagels. Pour cette dernière, en effet, l'orthodoxie spirituelle n'existe que par la mystification des Pères de l'Église, qui ont imposé la médiation de l'Église pour la connaissance du divin. La canonisation, pour Bruns, est l'affirmation du pouvoir de l'écrit détenu par les prêtres, aux dépens de la voix marginale des prophètes. Le canon ne fait qu'occulter l'exercice du pouvoir institutionnel en étouffant la dissidence. Tout comme le travail de Childs reprend une argumentation littéraire sur l'autorité intrinsèque du canon dans la tradition de T.S. Eliot et de la New Criticism, de même, les argumentations extrinsèques s'unissent aux tentatives de discréditer le canon littéraire. Il suffit de voir l'usage fréquent que fait Christine Froula des arguments de Pagels dans son offensive féministe contre l'idée même du canon littéraire comme répressive (FROULA 1983: 3236).

\section{Le canon: d'objet à procès}

L'essai de Frank Kermode sur Botticelli dans Forms of Attention est une étude originale et importante qui montre bien l'impossibilité de

"Contrary to the older idea that the New Testament, taken as a
whole, provides a balance and acts as a criterion which might prove
one of the forms of Christianity correct as against the others, it is
now increasingly considered to contain the seeds of a number of
different and potentially competing types of faith. There are
individual passages, strata, books or writers which may clearly
support one construction of Christianity as against another; but the
canon as a whole does not, Rather, it contains the seeds of
conflict.» (Bart 1983: 99)

12 C'est ce dernier point qui sous-tend l'insistance de Barr sur l'opposition entre la reconnaissance aveugle de l'autorité du canon et un respect constant pour «the acting persons who wrote some of it» et leurs «teachings, attitudes and actions».

13 Il est à noter que Barr reconnaît également cette possibilité dans la formation du canon (BARR 1983: 57-8). 
réduire le canon à une question de contenu intrinsèque ou de contraintes externes. Kermode souligne que les peintures qui avaient fait accéder Botticelli à la consécration du canon ont par la suite été reconnues comme n'étant pas de sa main, après que l'inclusion de Botticelli dans le canon ait fait porter sur ces oeuvres l'attention soutenue des experts. Et malgré cela, Botticelli est demeuré au panthéon de l'histoire de l'art... Cette anecdote illustre de manière évidente la fragilité de toute présomption d'une valeur intrinsèque du canon. Mais invalide également toute tentative de formuler les règles normatives de la canonicité et établit plutôt cette définition provisoire: le texte canonique est "un texte qui mérite une étude plus approfondie". C'est parce qu'un texte fait partie du canon qu'il est étudié, et non le contraire.

Le canon ne relève pas de règles formelles, puisque le texte canonique est, par définition, ce texte dont on étudie les règles. Kermode, dans "The Institutional Control of Interpretation", se réfère à l'exemple de la psychanalyse pour établir que toute exégèse, et donc, toute considération du canon, est à la recherche d'un "sens latent" (KERMODE 1979: 85). Nous reprendrons l'exemple de la psychanalyse pour réfuter ce que dit Kermode de la déconstruction, à savoir qu'à cause du fonctionnement nécessairement canonique de l'institution, la déconstruction ne serve qu'à donner une image novatrice au travail que les exégètes professionnels ont toujours fait (KERMODE 1979: 86). Bien au contraire, la déconstruction, de par sa réinscription du canon comme figure, et plus précisément, comme figure d'écriture, est en mesure de fournir une issue à l'impasse actuelle du débat sur le canon.

Interpréter le canon comme une figure d'écriture, c'est interpréter le canon à rebrousse-poil; c'est reconnaître que la canonicité déstabilise nécessairement toute forme figée que pourrait prendre un canon. Cette nécessaire instabilité n'est produite ni par un contenu changeant, ni par une règle formelle du canon, mais par une déconstruction "figurale" constitutive qui ne peut se réduire à un matérialisme historique ou à un formalisme esthétique. Dire que le fonctionnement du canon excède sa composition, c'est dire que ni la sociologie critique de Richard Ohmann, ni le relativisme comparatiste modéré de Lawrence Lipking ne peuvent épuiser le problème de la canonicité par une démystification sur des bases extrinsèques. Et dire que le canon ne peut être réduit à un formalisme conceptuel ou à un ensemble de règles détruit les défenses de l'autorité intrinsèque du canon -- qu'elles proviennent du positivisme impérialiste de T.S. Eliot ou de l'idéalisme libéral de Charles Altieri. 
Une description de la canonicité de la Bible, faite par Kermode luimême, fournit les éléments nécessaires à une lecture du canon comme figure. À l'article "The Canon" dans The Literary Guide to the Bible, Kermode indique que le remplacement progressif de la tradition orale par l'écriture a été le prélude nécessaire à l'établissement d'un canon (KERMODE 1987b: 602). Le canon biblique se forme lors du passage de l'oral à l'écrit. L'attention particulière qu'exigent les textes canoniques provient de leur qualité écrite : l'écriture accomplit une mise à distance qui à la fois stabilise un ensemble textuel et exige sa déstabilisation par des actes d'interpétation divers. Or, à cause de son rapport constitutif à l'écrit, le canon ne peut manquer de générer la transgression de ses propres limites par l'interprétation. Même lorsque l'écriture clôt ou fixe le canon, elle demande à être interprétée. Et comme le canon est explicitement écrit plutôt qu'oral, cette interprétation est nécessairement ouverte plutôt que fermée: l'interprétation ne peut être contrôlée par référence à une origine première parce que cette origine serait orale et donc, en soi, non canonique. L'articulation de l'cauto-transgression" du canon avec la question de l'écriture est particulière à la culture biblique. Mais même dans des canons non textuels, (tels les collections d'objets sacrés ou les vins d'appellation contrôlée), Joseph $Z$. Smith a pu identifier le même mouvement simultané de fondation et de suspension de l'origine caractéristique du fonctionnement biblique. Smith voit le canon comme une liste finie, close, qui demande l'habileté d'un herméneute pour appliquer sa règle et interpreter "everything that is known or everything that is" (SMITH 1978: 23). La figure du canon se présente comme une figure maîtresse qui permet d'analyser l'auto-transgression inévitablement générée par la culture: à la fois invocation de la nature ou de l'origine, et suspension de la possibilité de les retrouver. Le rapport entre l'écrit et l'oral apparaît dans cette perspective comme l'instance canonique particulière à la culture occidentale. Plus qu'une simple coïncidence d'intérêts pour le rapport oral/écrit, cette question constitue une intersection significative entre la déconstruction et le canon (voir Derrida, De la grammatologie). Les défenses intrinsèques et les démystifications extrinsèques du canon sont aussi contraignantes que les répressions effectuées au nom du droit canon, parce qu'elles refusent de voir que tout canon comporte nécessairement sa propre transgression.

Le canon est ce qui est écrit, préservé de l'instabilité et de la fragilité de l'oral. Cependant, l'écriture transgresse radicalement la stabilité et la fermeture que présente le canon, en demandant à être interprétée. Si le canon est ce qui est écrit, l'écriture elle-même 
semble impuissante à prendre place dans le canon: il y a, dans l'écriture, quelque chose de radicalement non canonique -- d'apocryphe. Kermode signale, dans l'étymologie du mot apocrypha, une contradiction qui indique bien la manière dont la canonicité est à la fois produite et suspendue par l'écriture. Le sens de apocrypha se modifie, de "texte réservé à l'usage du sage", tel qu'employé par exemple par Esdras dans l'Ancien Testament, à la signification de textes exclus parce que faux ou d'origine incertaine. Le fait de sélectionner des textes apparaît comme une activité à double tranchant, qui détruit ou brouille l'opposition entre le canonique et l'apocryphe. Si le mot grec "apocryphe" signifie ce qui est caché, obscur, et difficile à comprendre, c'est précisément ce qui demande à être interprété. D'autre part, si le canon est ce qui demande à être interprété, le canon est lui-même apocryphe ${ }^{14}$. Et ce qui est caché, apocryphe dans le canon, est un certain système de signes ou de traces, une cryptographie -- l'écriture.

\section{La psychanalyse, l'écriture et le canon}

La psychanalyse freudienne est peut-être la plus importante réflexion moderne sur les rapports d'interdépendance entre la culture et le canon au XXe siècle. L'intérêt de la psychanalyse ne tient pas à ce qu'elle peut nous apprendre de certains textes particuliers, mais plutôt au fait qu'elle montre le canon comme une figure d'écriture inhérente à la problématique de la culture occidentale. L'édition canonique des écrits de Freud dans la Standard Edition anglaise est issue de la disciplinarité particulière de la psychanalyse .- paradoxalement, une discipline qui promet de démystifier certaines idées reçues (dont la canonicité biblique) en même temps qu'elle insiste sur sa canonicité particulière. La figure de Freud, à cause du clivage fécond de son projet psychanalytique, est capitale pour cette étude. Freud tente à plusieurs reprises d'installer la psychanalyse dans une épistémologie "scientifique", alors qu'il est étudié avec application par ceux qui, en arts et en sciences humaines, sont les plus déterminés à déstabiliser l'autorité univoque du savoir produit par la méthode scientifique. Autrement dit, l'écriture de Freud est à la fois laïque et canonique. Elle reproduit, bien malgré elle, le canon comme clôture et forclusion.

14 Kermode souligne également que c'est le propre du texte canonique que de «souiller les mains" (KERMODE 1978b: 601), ce qu'en bons post-platoniciens, nous aurions plutôt tendance à attribuer au texte apocryphe. Le canon n'est pas dépositaire de la pureté mais bien de la contamination. Le travail de l'interprétation reste toujours à faire et, en tant que travail, exige une lecture plutôt qu'une croyance. 
Cette subversion d'elle-même découle du fait que la psychanalyse est une entreprise "scientifique" fondamentalement obsédée par sa propre canonicité disciplinaire; or cette obsession prend une forme très "littéraire".

Parlant de stades de développement humain, de développement des abstractions intellectuelles, d'étymologie, la psychanalyse se réclame d'une scientificité historique dans ses descriptions du développement individuel, institutionnel et linguistique. À cet égard, l'analyse de Freud dans Moïse et le monothéisme s'apparente à la tradition historiciste de critique biblique. En même temps, une psychologie de la structure intrinsèque de la subjectivité devient une discipline canonique légitimée précisément par le sacrifice qu'elle fait de toute prise extérieure sur le savoir positif. Dès lors, le seul sujet d'étude possible pour Freud sera Freud lui-même. La psychanalyse aura des visées tant extrinsèques qu'intrinsèques, et cherchera à démystifier et canoniser tout à la fois. Le retour obsessionnel aux problèmes d'écriture révèle la canonicité présente dans la science qui se veut "profane" de Freud. L'écriture, pour lui, est la suspension malaisée et interminable d'une origine non représentable: I'Inconscient. Ce qui revient à dire que l'Inconscient est le modèle de canon le plus important du XXe siècle.

Pour illustrer la lecture littéraire du canon biblique, nous considérerons Moïse et le monothéisme de Freud. Le titre original prévu par Freud en était: "Moïse : un roman historique", ce qui indique assez clairement que la lecture freudienne du récit biblique soit à la fois littéraire et psychanalytique (il serait plus correct de dire littéraire parce que psychanalytique).

Freud réinterprète l'histoire de Moïse pour exposer le mélange de superstition et de mythe produit par la canonisation; il promet de retrouver scientifiquement l'histoire réelle qui, jusqu'à présent, nous a été illisible à cause du respect accordé au canon, dépositaire des valeurs religieuses ${ }^{15}$. Les critiques contemporains du canon littéraire ont proposé, de façon très similaire, de retrouver l'histoire réelle d'oppression et d'exclusion que nous ne pouvions lire à cause du respect accordé au canon comme dépositaire du mérite littéraire.

15 «... we cannot allow any such reflection to induce us to put the truth aside in favour of what are supposed to be national interests, and, moreover, the clarification of a set of facts may be expected to bring us a gain in knowledge» (FREUD 1939: 7). 
Freud découvre que Moïse était un Égyptien qui est mort assassiné par le peuple juif. La canonisation biblique a été une tentative de dissimuler les traces de ce crime: "In its implications the distortion of a text resembles a murder: the difficulty is not in perpetrating the deed, but in getting rid of its traces" (FREUD 1939: 55). L a canonisation ressemble au crime (le meurtre) qu'elle tente de dissimuler. Des traces anciennes du corps du délit toujours présentes dans le texte se manifestent comme autant d'incohérences et d'ambiguïtés. Freud se propose de guérir le texte de sa maladie canonique, en révélant scientifiquement une vérité historique enfin libérée des traces archaïques du mythe, que la canonisation avait occultée.

Pour Freud, l'histoire canonique est une distorsion d'événements historiques réels. Le discours de l'histoire doit être distingué du reliquat mythique présent dans la narration comme les traces de l'Inconscient dans le discours du patient. Ainsi le travail de Freud «leads to a conclusion which reduces religion to a neurosis of humanity and explains its enormous power in the same way as a neurotic compulsion in our individual patients" (FREUD 1939: 55). Dans la Bible, l'Histoire est malade de l'infection mythique et superstitieuse transmise par la canonisation. Analysis Terminable and Interminable de Freud établit un parallèle explicite entre la persistance de la névrose et celle de la superstition: "Of all the erroneous and superstitious beliefs of mankind that have supposedly been surmounted, there is not one whose residues do not live among us today..." (FREUD 1937: 229). Tout comme le traitement d'une névrose requiert une transformation du moi, le traitement de la Bible exige d'éliminer les distorsions mystificatrices de l'histoire. Le statut canonique de la Bible ("reverence for Biblical tradition" (FREUD 1939: 9)) est une superstition qui a conduit à accepter de manière erronée un mythe comme vérité historique.

Freud tente, dans Moïse et le monothéisme, de démystifier le canon et dexposer au grand jour une mystification raciale. Cette tentative survient au moment même de l'invasion de l'Autriche par les nazis, alors que la démystification de certains autres mythes racistes aurait été autrement plus pressante. Pourquoi ce texte représente-t-il plus qu'un épisode malencontreux dans le développement de la critique historique de la Bible? Le texte de Freud est remarquable parce qu'il met constamment en relief la figure de la canonicité dans sa lecture de la Bible, le texte canonique par excellence. Plutôt qu'à une simple contradiction interne, cette lecture du canon comme figure mène à 


\section{Bill Readings: Le canon et ses suites}

quelque chose de beaucoup plus intéressant. La lecture psychanalytique du canon est plus littéraire que scientifique, probablement parce que la psychanalyse accorde beaucoup d'importance au caractère narratif dans sa propre visée "scientifique". En ceci, elle demeure plus ouverte au littéraire que la plupart des lectures du canon produites en études littéraires ou à leur encontre.

Même si Freud, au nom de la science, vise à faire disparaître un respect superstitieux pour le canon, à remplacer un récit mythique par le discours scientifique, le fil de sa propre écriture demeure troublé par le reliquat archaïque d'une névrose. De la même manière que la canonisation, qui ne parvient pas à faire disparaitre les traces du crime, ressemble au meurtre qu'elle tente de camoufler, l'écriture de Freud ressemble au mythe canonique qu'elle tente de dissiper. Le texte freudien est plein des mêmes "écarts très notables, répétitions troublantes et contradictions évidentes" qu'il trouve dans le texte canonique, révélant ainsi “des choses qu'il ne devait pas nous communiquer" (FREUD 1939: 43). Ainsi son propre texte comporte précisément les défauts ou anomalies (répétitions, coupures inopinées dans le récit, enchâssement de matériaux étrangers, contradictions) qui amènent Freud à suggérer que tout n'est pas parfaitement sain dans le texte biblique. Le texte freudien déclare son échec, morcelle le travail en trois essais avec de multiples subdivisions et confesse l'interruption d'une analyse pour la reprendre à trois reprises ${ }^{16}$. Par ailleurs, quelque chose qui ne peut qu'être qualifié de "piété superstitieuse" (FREUD 1939: 43) conduit Freud à conserver son texte au point de garder dans sa forme originale la première note de préface, que des circonstances nouvelles rendent incongrue. Une préface générale aurait pu aisément rendre compte du retard de publication. Freud thésaurise donc son texte et va jusqu'à reproduire dans son propre texte la piété superstitieuse entourant le texte sacré, imitant les répétitions qui sont censées

16 «... it will therefore be better to leave unmentioned any further implications of the discovery that Moses was an Egyptian [FREUD1939: 16] ... Now, however, or so it seems, our work has reached a provisional end. For the moment we can draw no further conclusions from our hypothesis that Moses was an Egyptian whether it has been proved or not [FREUD 1939: 33] ... we shall have to admit that the thread which we have tried to spin from our hypothesis that Moses was an Egyptian has broken for the second time. And this time, as it seems, with no hope of mending [FREUD 1939: 36] ... And here, it seems I have reached the conclusion of my study, which was directed to the single aim of introducing the figure of an Egyptian Moses into the nexus of Jewish history [FREUD 1939: 52]». Toutes ces citations proviennent des deux premières parties, qui font moins de la moitié du texte -- apparemment. 
signaler les nombreux auteurs de la Bible lorsqu'il récapitule entièrement son livre dans la troisième section.

L'écriture de Freud est dérangée, incommodée par le même problème qu'elle diagnostique dans la Bible: des reliquats archaïques se manifestant comme des perturbations dans le texte. Les symptômes de la présence de perturbations textuelles sous l'égide de la double pulsion de conservation et de changement, qui structure la canonisation, sont mises en scène dans l'écriture. L'analyse de Freud propose de réduire la tradition autoritaire de la Bible à son seul élément d'histoire scientifique. Or cette autorité "scientifique" est acquise au prix de l'infection de l'analyse scientifique de Freud par tous les symptômes de la maladie du canon qu'il propose de guérir ${ }^{17}$.

Dans cette histoire d'un grand homme, de Moïse l'homme, fondateur d'une tradition intellectuelle contre lequel se sont rebellés ses disciples, Freud retrace les débuts du mouvement psychanalytique et ses rapports avec ses disciples. Freud fait référence à l'apostasie de Rank (“ln 1909 Otto Rank, who was at that time still under my influence" (FREUD 1939: 10)) en des termes qui l'obligent à fournir une note de bas de page préventive qui pourrait sembler superflue à un lecteur inconscient de ce qui est en jeu pour Freud : "it is far from being my intention to belittle the value of Rank's independent contributions to the work" (FREUD 1939: 10n). Son intention dans ce passage semble obscure, à moins qu'il ne s'agisse de déplorer le reniement dont il a été lui-même victime. Une analogie entre l'histoire des débuts du peuple juif et l'histoire des débuts de la psychanalyse prend forme à travers les remarques de Freud au sujet de sa propre captivité en Autriche et son désir d'éviter une autre diaspora.

Redondant, contradictoire et troublé, le texte de Freud prétend dissiper les perturbations inscrites dans le texte canonique pour y lire l'histoire d'une fronde turbulente des fils contre le père, qui est également l'histoire du mouvement psychanalytique dont il est le père et le "grand homme". Dans l'histoire de la psychanalyse, après la destruction du temple viennois lors de l'Anschluss, une certaine attention à l'écriture, à la qualité littéraire du canonique, se fait jour.

17 Michel de Certeau, dans Le roman freudien, a foumi une description plus ample des liens complices entre psychanalyse, littérature et historiographie scientifique, sans toutefois reconnaître plus de rigueur au champ littéraire que celle d'une vague hétérogénéité. 
“Immediately after the destruction of the Temple in Jerusalem by Titus, the Rabbi Jochanan ben Zakkai asked permission to open the first Torah school in Jabneh. From that time on, the Holy Writ and intellectual concern with it were what held the scattered people together" (FREUD 1939: 115). Ici encore, le grand démystificateur scientifique du canon reproduit la figure de la canonicité au sein de sa propre écriture.

\section{Le canon comme figure: l'écriture et la déconstruction}

L'amorce d'une lecture déconstructive de ce texte de Freud nous a permis de voir le canon, à cause de son rapport complexe à l'écriture, comme une figure inconcevable en termes d'intériorité ou d'extériorité. L'écriture, geste qui semble devoir déterminer l'intériorité du canon, circonscrire ses marges, travaille le canon. Elle le hante comme une figure elle-même supplémentaire à l'intériorité circonscrite par l'écriture. L'écriture, tout comme le canon, déstabilise nécessairement l'opposition intériorité/extériorité par le biais de laquelle elle est déterminée. En reconnaissant que le canon est une figure qui trouble la dichotomie intérioritél extériorité, nous redéfinissons de manière féconde les termes des assertions de valeur intrinsèque ou des critiques extrinsèques qui ont jusqu'à présent déterminé l'essentiel du débat sur le canon.

À travers tout ce débat, les auteurs, même ceux aussi soucieux de représenter l'ensemble des positions sur cette question que Barbara Herrnstein Smith, ont eu tendance à ne songer qu'assez distraitement à l'impact éventuel de la déconstruction sur la conception du canon. II nous semble possible que la manière dont les termes du débat sur le canon sont déstabilisés par une lecture déconstructive de Freud puisse, jusqu'à un certain point, expliquer cette lacune. L'analyse déconstructive du canon doit résister à la polarisation simpliste des descriptions du canon fondées sur les arguments intrinsèques ou extrinsèques, qui, nous l'avons vu, structure en général ce débat. Il est nécessaire d'insister plutôt sur l'hétérogénéité "figurale" du canon par rapport à tout savoir dont la prétention serait de se situer confortablement à l'intérieur ou à l'extérieur du canon. Le canon devient ainsi une sorte de structure 
"parergonale", dérangeant l'opposition entre "son" intériorité et "Son" extériorité (voir "Le Parergon" de Jacques Derrida ${ }^{18}$ ).

Le canon écrit est miné par sa propre écriture. L'injustice commise au cours des siècles au nom du canon est le produit d'un oubli délibéré de la nature figurale du canon et l'assertion d'une essence du canon. Le canon à la fois permet et empêche la production des textes. Lire le canon à rebrousse-poil, c'est mettre en question un consensus superstitieux qui renforce l'injustice, tout en reconnaissant que la tentation de démystifier ne fait toujours appel qu'à un autre consensus superstitieux, celui du mythe du dévoilement de la vérité, que l'on découvrirait toute nue. Lire le canon comme une figure ne serait pas le nier, mais le reconstruire comme inévitablement toujours inadéquat. $\mathrm{Ce}$ n'est que de cette façon que le canon peut devenir un site de résistance.

Dans Moïse et le monothéisme, les turbulences de la démystification "scientifique" du canon sont toujours liées à des traces laissées par Freud lui-même, indiquant les difficultés de son projet d'écriture. Pour résumer, ces traces signalent, d'une part, le caractère forcément hypothétique de ce savoir, d'où le recours à une technique narrative plutôt qu'à une description scientifique; elles évoquent, d'autre part, les nombreuses difficultés pratiques de la situation de Freud écrivant dans un contexte politique et culturel donné. Comme un criminel revenant sur la scène du crime. Freud ne cesse de retourner à la scène de l'écriture, scène divisée tout comme la figure du canon. Freud admire Darwin parce qu'il a ébranlé l'édifice de la religion mais fournit, comme l'une des preuves éclatantes du succès de Darwin, sa canonisation dans la sphère même de la piété et de la superstition:

Let us take, for instance, the history of a new scientific theory, such as Darwin's theory of evolution. At first it met with embittered rejection and was violently

18 J. Derrida, La vérité en peinture. Le canon partage avec la trace de l'écrit cette «infrastructuralité» parergonale (selon le mot de Rodolphe Gasché), comme le montre Derrida dans De la dissémination. Le terme d'«infrastructure» est repris par Gasché pour décrire la série des éléments ou des anti-concepts déconstructifs tels la trace, l'hymen, le pli, le pas, etc., qui servent à la fois dans le texte derridien à constituer et à défaire les oppositions binaircs, fonctionnant comme autant de troisièmes termes non dialectiques, recouvrant toujours les deux opposés et jamais aucun à la fois. L'observation du déplacement parergonal des concepts par la trace écrite peut être comprise comme le procédé déconstructif de base : il est en effet plus largement disséminé dans le texte derridien que la dissémination elle-même. 
disputed for decades; but it took no longer than a generation [40 années au désert!] for it to be recognized as a great step forward towards truth. Darwin himself achieved the honour of a grave or cenotaph in Westminster Abbey. A case such as this leaves us very little to unravel (FREUD 1939: 66-7).

Un exemple comme celui-ci est d'un illogisme si criant dans son recours à l'argument per auctoritatem, utilisant pour sa cause la chose même qu'il vise à ébranler, qu'il semble, indeed, laisser "very little to unravel». Ne demeure plus que la figure de la canonicité, apparaissant encore et toujours comme un appel à la scène parergonale de l'écriture. La démystification aura atteint son but une fois qu'elle aura elle-même été canonisée, c'est-à-dire mystifiée. L'écriture sert à protéger un savoir des périls de l'histoire, mais elle délégitimise nos prétentions à la science en activant la figure du canon. La scène parergonale de l'écriture qui est présente dans le texte de Freud fonctionne en parallèle avec les reliquats archaïques de superstition que Freud s'était juré d'exorciser du canon biblique. La cure est elle-même maladive, elle n'est en fait que le symptôme de l'infection, et requiert un traitement toujours à reprendre, le retour en analyse de l'analyste que propose Freud dans Analysis Terminable and Interminable.

Freud parle dans ce texte de trois domaines professionnels "impossibles": l'enseignement, la politique et la psychanalyse (FREUD 1937: 248). L'analyse est impossible parce que l'idée qu'une guérison est complète est le signe qu'une analyse plus poussée est nécessaire : la conclusion dans ce cas-ci est toujours illusoire. L'enseignement, la politique et la littérature exigent une même attention au fait que tout fantasme de conclusion est une forclusion -- au mieux, un refoulement. Nous voilà revenus à la circularité épistémologique des arguments canoniques, selon lesquels le canon détermine l'échelle des valeurs de ceux qui prétendent déterminer la valeur du canon. Ceci ne veut pas dire, cependant, que nous devons jeter le canon par-dessus bord pour adopter une sociologie comparatiste des valeurs culturelles. Comme notre lecture de Freud l'a montré, toute prétention de scientificité sera traversée par la figure de la canonicité. Il est impossible de perpétuer un canon comme le voudraient ses plus véhéments apologues, c'est-àdire comme dépositaire de l'étalon des valeurs collectives. 


\section{Conclusion}

Le point de départ de cette critique du canon doit être précisément une méfiance vis-à-vis de la prétention de conclure une fois pour toutes, que ce soit en matière de valeurs esthétiques ou d'organisation politique : cette prétention masque toujours une oppression. Cette lecture doit aussi nous faire prendre conscience du fait que l'histoire ne peut jamais être expurgée des traces archaïques du mythe. Même si la science historique promet cette épuration, même si les critiques du canon promettent d'éliminer les traces de superstition littéraire, il faut bien voir que ce processus ne peut jamais être complété. Croire en la possibilité d'en finir un jour avec le canon entraîne nécessairement un retour en analyse du critique, un questionnement de l'aveuglement qui aurait permis cette croyance. Si l'histoire est un comple rendu écrit de ce qui s'est vraiment passé, maintenant à distance l'élément mythique de la tradition orale, l'événement de l'écriture partage de la même nonscientificité du mythe que la mise à l'écrit de la tradition voulait éliminer.

Le caractère clos du canon littéraire a fait un objet de savoir de la littérature en occultant l'écriture, ce qui a favorisé plusieurs gestes de répression politique, dont les plus évidents sont ceux des femmes et des minorités. Toutefois, le rejet pur et simple du canon est un rejet de l'écriture au nom de la Raison. Le bannissement de la littérature au nom du savoir est un mythe répressif qui remonte à La République de Platon. Le canon n'est pas un mythe qui puisse être éliminé, non pas parce que nous aurions besoin des valeurs dont il serait porteur, mais parce que le canon, en tant qu'écriture, (se) résiste (à) lui-même. Et cette résistance (à soi), dans la mesure où elle mine la possibilité d'identité, ne peut servir à fonder une nouvelle intériorité négative par laquelle les textes canoniques seraient résistants à/en eux-mêmes. La figure du canon dérange nécessairement toute intériorité et du même coup la possibilité de valeurs purement intrinsèques. Le canon est par définition écrit, mais l'écriture figure dans le canon comme la trace du processus primaire inconscient dans un discours secondaire conscient. Le canon n'est pas seulement une liste d'oeuvres conservées et transmises pieusement, pas plus qu'il ne doit être une nouvelle liste d'oeuvres, réformée au nom de visées particulières. La canonicité est un processus divisé, comme l'écriture, contre elle-même. Les discussions au sujet de ce qui devrait faire, ou non, partie des canons, ou quant à savoir s'il 
devrait même y avoir un canon, ne sont pas près de se terminer. Mais elles n'épuisent pas la question de la canonicité et tendent plutôt, en fait, à étouffer dans l'oeuf une réflexion sur ce que peut signifier la lecture. Une étude du canon biblique montre la nécessité de considérer attentivement le passage de l'oral à l'écrit. Le statut divisé de l'écriture, la manière dont le geste de l'écriture suspend radicalement la fixité canonique de l'écrit, est ce qui donne au débat sur le canon son importance et permet d'entrevoir le véritable travail de la lecture.

Le canon littéraire n'est pas dépositaire de l'étalon des valeurs littéraires: il est composé de textes qui demandent à être relus et qui, en ce sens, sont littéraires. Le canon biblique ne contient pas la vérité transparente: il pose, à cause de sa clôture même, l'exigence d'une exégèse et d'une application. Que la lecture soit guidée par l'Église (catholicisme) ou par l'Esprit (protestantisme), la nature, les limites, et la veracité de toute interprétation normative ou officielle doit ellemême être établie par des actes d'interprétation supplémentaires (l'autorité de l'Église se fonde sur une interprétation d'une phrase de Jésus, la question de savoir si l'on est guidé par l'Esprit ou par le diable en guise d'ange dans sa fidélité absolue au texte de la Bible tourmente les protestants les plus fondamentalistes). Par l'admission que ces textes doivent nécessairement être relus encore une fois, que leur lecture est à la fois nécessaire et impossible (qu'elle ne peut être conclue ou épuisée), la canonicité nie que l'écriture puisse être épuisée, saisie, par ce qui est écrit; nie la présomption que l'écriture devienne un savoir. Le canon comme concept réduit nécessairement la textualité à une question de connaissance, qu'il s'agisse du savoir intrinsèque de la valeur ou de la vérité, ou du savoir extrinsèque de l'idéologie ou des erreurs de recension. Dans la mesure où il prétend énoncer des prescriptions en rapport avec un état de choses qui puisse être décrit exhaustivement, c'est-à-dire comme objet de savoir, le canon est indéfendable contre l'assertion que son "universalité" doit nécessairement exclure quelqu'un. Une lecture du canon comme figure nous rappelle que l'importance du canon ne tient pas un savoir ou à une valeur contenus en lui, mais plutôt au fait que sa figure interdit au savoir d'oublier l'événement de son inscription. La figure de la canonicité revient sans cesse comme écriture et nie que la compréhension de la culture à travers l'étude de son canon puisse être la révélation d'une origine, extrinsèque (historique) ou intrinsèque (fonctionnelle). L'autotransgression canonique signale l'impossibilité constitutive d'atteindre un jour une instance légitimant la représentation: elle suspend indéfiniment l'origine en faveur d'une altérité. 
Les auteurs précités, JanMohamed et Froula en littérature, tout comme Pagels en études religieuses, sont bien fondés d'indiquer la violence répressive de l'exclusion qui est à l'oeuvre dans toute tentative d'établir le concept du canon comme expression d'un héritage culturel universel. Nous devons aussi tenir compte des nombreux avertissements de Frank Kermode et Jonathan Smith à l'effet que la représentation d'une culture est elle-même un exemple de canon. Le canon, par définition clos, demande à être ouvert et appliqué, ce qui exige une ingéniosité certaine. Découvrir par le biais de la lecture, c'est, selon l'étymologie latine, invenire, c'est-à-dire à la fois apprendre et inventer. La lecture est à la fois une exécution fidèle et une construction habile. Ces deux versants de la lecture peuvent se combiner dans la prise de conscience déconstructiviste, issue d'une lecture de la psychanalyse en tant que canon, que l'écriture comme figure au sein de la représentation marque l'impossible simultanéité de l'invocation de l'origine et sa suspension. L'écriture est ce qui constitue le canon occidental: l'impossible et nécessaire dépendance des représentations culturelles face à une origine (le Verbe divin, la Nature, la tradition, I'Inconscient) est radicalement irreprésentable. Le canon signale jusqu'à quel point la culture découle de l'impossibilité de représenter l'origine. L'écriture marque pour l'Occident le degré auquel la représentation dépend d'une "secondarité". Le canon n'est pas le souvenir pieux d'une origine, mais la trace de l'immémorial: ce qui ne peut être oublié mais ne peut être pleinement présent en mémoire. En ce sens, et contrairement au christianisme biblique, le canon est nécessaire à la culture alors que l'origine ne l'est pas. Les descriptions extrinsèques du canon trouvent son origine "derrière" le concept; les descriptions intrinsèques conçoivent le canon comme origine. Mais le canon n'est pas un concept: en faire l'objet d'une représentation, c'est nier qu'il évoque l'irreprésentable. Le canon exige à la fois fidélité et invention, et c'est dans l'espace entre indigence et fécondité que prennent place le commentaire de la lecture et de la culture. Le canon doit être lu comme l'arc d'un trope, qui suspend radicalement l'origine et constitue paradoxalement la possibilité et l'impossibilité d'une représentation immanente de la culture, l'autoprésence de la culture à elle-même. 


\section{Bill Readings: Le canon et ses suites}

\section{Bibliographie}

ALTIERI, Charles. 1983. "An Idea and Ideal of a Literary Canon", Critical Inquiry 10/1.

BARR, James. 1983, Holy Scripture: Canon, Authority, Criticism. Philadelphia, Westminster Press.

BRUNS, Gerald. 1984. "Canon and Power in Hebrew Scriptures", Critical Inquiry 10/ 3.

CHILDS, Brevard, 1986. Old Testament Theology in a Canonical Context. Philadelphia, Fortress Press.

De CERTEAU, Michel. 1985. "The Freudian Novel", dans Heterologies. Minneapolis, University of Minnesota Press.

DERRIDA, Jacques. 1967. De La Grammatologie. Paris, Minuit.

IDEM. 1967. L'écriture et la difference. Paris, Minuit.

IDEM. 1972. La dissémination. Paris, Seuil.

IDEM. 1978. La vérité en peinture. Paris, Flammarion.

ELIOT, Thomas Stearns. 1960."Tradition and the Individual Talent", dans The Sacred Wood. London, Methuen.

IDEM. 1964. The Use of Poetry and the Use of Criticism. London, Faber and Faber.

FREUD, Sigmund. 1910. "Leonardo Da Vinci and a Memory of his Childhood" dans The Standard Edition of the Complete Psychological Works of Sigmund Freud. (Strachey et al., trad.), Tome II, Londres, Hogarth Press.

IDEM. 1937. "Analysis Terminable and Interminable" dans The Standard Edition of the Complete Psychological Works of Sigmund Freud. (Strachey et al.,trad.), Tome XXIII, Londres, Hogarth Press.

IDEM. 1939. "Moses and Monotheism" dans The Standard Edition of the Complete Psychological Works of Sigmund Freud. (Strachey et al., trad.), Tome XXIII, Londres, Hogarth Press.

FROULA, Christine. 1983. "When Eve Reads Milton: Undoing the Canonical Economy", Critical Inquiry 10/2.

GASCHÉ, Rodolphe. 1988. The Tain of the Mirror . Cambridge, Mass., Harvard University Press.

GUILLORY, John. 1983. "The Ideology of Canon Formation", Critical Inquiry $10 / 1$.

HERRNSTEIN SMITH, Barbara. 1983. "Contingencies of Value", Critical Inquiry 10/1.

JANMOHAMED, Abdul. 1985. "The Economy of Manichean Allegory: The Function of Racial Difference in Colonialist Literature", Critical Inquiry 12/1. 
KERMODE, Frank. 1979. "Institutional Control of Interpretation", Salmagundi 43.

IDEM. 1987a. Forms of Attention. Chicago, University of Chicago Press. IDEM. 1987b. "The Canon", dans The Literary Guide to the Bible. (ALTER, Robert \& Frank KERMODE, ed.), Cambridge, Mass, Harvard University Press.

KRUPAT, Arnold. 1983. "Native American Literature and the Canon", Critical Inquiry 10/1.

LIPKING, Lawrence. 1983. "Aristotle's Sister:A Poetics of Abandonment", Critical Inquiry 10/1.

LYOTARD, Jean-François et Jean-Loup THÉBAUD. 1979. Au juste. Paris, Christian Bourgeois.

OHMANN, Richard. 1983. "The Shaping of a Canon: US Fiction 1960-65", Critical Inquiry 10/1.

PAGELS, Elaine. 1979. The Gnostic Gospels. New York, Random House.

ROBINSON, Lillian. 1985."Treason Our Text: Feminist Challenges to the Literary Canon", dans The New Feminist Canon. (SHOLWATER, Elaine, ed.), New York, Pantheon.

SMITH, Joseph Z. 1978. "Sacred Persistence : Towards a Redescription of Canon", dans Approaches to Ancient Judaism: Theory and Practice. (GREEN, W.S., ed.), Missoula, Scholars Press.

SPIVAK, Gayatri. 1987. In Other Worlds. New York, Methuen. 